\title{
Microelectromechanical systems vibration powered electromagnetic generator for wireless sensor applications
}

Received: 1 August 2005/ Accepted: 28 October 2005/Published online: 25 April 2006

(C) Springer-Verlag 2006

\begin{abstract}
This paper presents a silicon microgenerator, fabricated using standard silicon micromachining techniques, which converts external ambient vibrations into electrical energy. Power is generated by an electromagnetic transduction mechanism with static magnets positioned on either side of a moving coil, which is located on a silicon structure designed to resonate laterally in the plane of the chip. The volume of this device is approximately $100 \mathrm{~mm}^{3}$. ANSYS finite element analysis (FEA) has been used to determine the optimum geometry for the microgenerator. Electromagnetic FEA simulations using Ansoft's Maxwell 3D software have been performed to determine the voltage generated from a single beam generator design. The predicted voltage levels of $0.7-4.15 \mathrm{~V}$ can be generated for a two-pole arrangement by tuning the damping factor to achieve maximum displacement for a given input excitation. Experimental results from the microgenerator demonstrate a maximum power output of $104 \mathrm{nW}$ for $0.4 \mathrm{~g}\left(\mathrm{~g}=9.81 \mathrm{~m} \mathrm{~s}^{-1}\right)$ input acceleration at $1.615 \mathrm{kHz}$. Other frequencies can be achieved by employing different geometries or materials.
\end{abstract}

\section{Introduction}

The rapid increase in demand for remotely located wireless devices has demonstrated the need for self-sufficient power sources. The growth in wireless sensor networks has been greatly facilitated by developments in low-power communications standards such as Zigbee and IEEE 802.15.4. Similarly, low-power microelectro-

E. Koukharenko $(\bowtie) \cdot$ S. P. Beeby $\cdot$ M. J. Tudor $\cdot$ N. M. White School of Electronics and Computer Science,

University of Southampton, SO17 1BJ Southampton, UK

E-mail: ak@ecs.soton.ac.uk

T. O’Donnell $\cdot$ C. Saha $\cdot$ S. Kulkarni $\cdot$ S. Roy

Tyndall National Institute, Prospect Row, Cork, Ireland mechanical systems (MEMS) have allowed the development of highly accurate, portable sensors and instruments for numerous applications in health care, condition monitoring (embedded sensors in building and bridges), consumer products, and defence [1]. Wireless operation enables such microsystems to be completely embedded in a structure with no physical connection to the outside. Typically, the energy necessary to power such systems is stored in batteries, which have drawbacks: they tend to be bulky, contain a finite amount of energy and have a limited shelf life. The replacement of batteries places an additional cost and environmental burden on the use of wireless sensor systems and is not possible in embedded applications. Alternative power sources can potentially overcome these drawbacks.

A potential alternative to batteries is miniature, selfcontained, renewable power supplies that convert energy from an existing source in the environment into electrical energy. The most common ambient sources are solar, vibration and thermal. In this work, we have used the principle of electromagnetic induction for the generation of electrical energy from kinetic energy present in the environment in the form of vibrations [2-5]. An earlier device developed at the University of Southampton was around $3,000 \mathrm{~mm}^{3}$ in volume and was fabricated using conventional machining techniques [6]. The motivation for this work is the development and fabrication of a MEMS version of this generator that uses standard silicon-based fabrication techniques and a low-cost, batch process.

\section{Microgenerator structure}

The arrangement of the MEMS version of the device, based on four magnets, is shown in Fig. 1. The coil is recessed in a silicon cantilevered paddle designed to vibrate laterally in the plane of the wafer. Discrete magnets are positioned within etched recesses in capping wafers that are bonded to each face of the middle wafer. The bonding process is aligned to ensure correct place- 


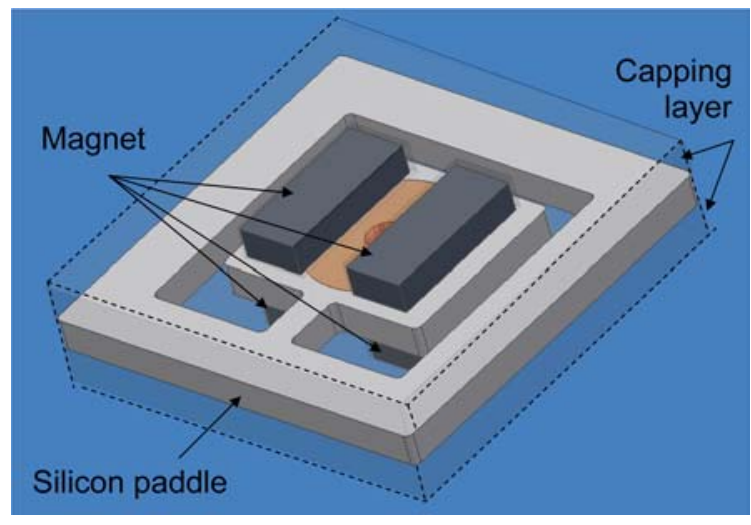

Fig. 1 Micromachined silicon generator

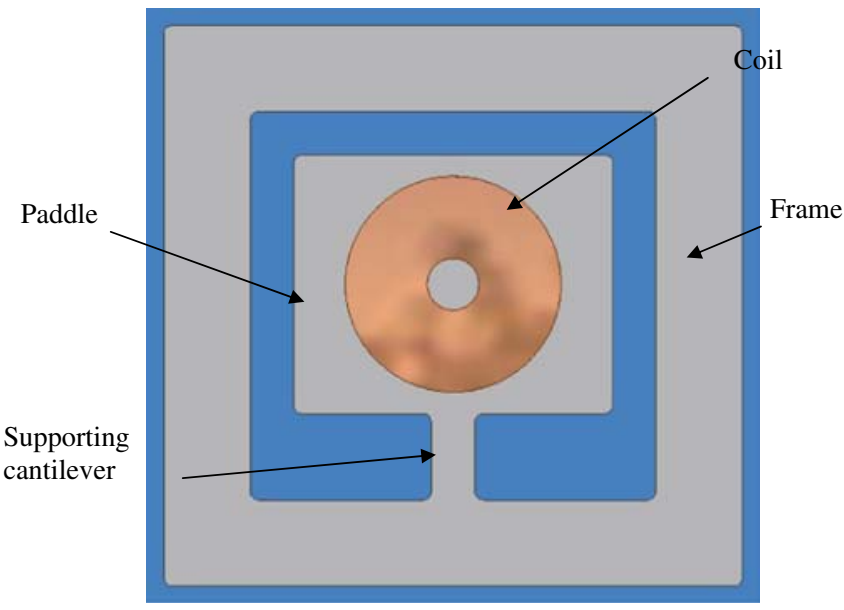

Fig. 2 Top view silicon paddle

ment of the coil relative to the magnets. Figure 2 shows the top view of the paddle layer, which is fabricated by deep reactive ion etching (DRIE) through the total thickness of the wafer.

\section{Microgenerator mechanical modelling}

The mechanical characteristics of the moving component of the generator (the cantilevered paddle) have been simulated using ANSYS finite element analysis (FEA). The natural frequencies of different cantilever configurations machined in silicon have been determined using modal analysis. Mechanical stresses induced by the deformation have also been simulated in order to ensure that the structure can withstand repeated cyclical stressing at maximum amplitude.

Three sets of supporting paddle beam dimensions have been simulated, each $1 \mathrm{~mm}$ long and $500 \mu \mathrm{m}$ thick. In model $A$ the beam is $500 \mu \mathrm{m}$ wide, model $B 400 \mu \mathrm{m}$ wide and $C 300 \mu \mathrm{m}$ wide. Table 1 lists the resonant frequencies of the fundamental lateral modes of the generator, which is the operating mode of interest. This mode is shown viewed from above in Fig. 3 .
Table 1 Simulation results for the natural frequency, $f_{\text {res }}$, voltages, $V_{\text {load}}$, (peak) and power, $P_{\text {load }}$, delivered to the load resistance, $R_{\text {load }}$ for three generator structures with $500 \mu \mathrm{m}$ separation distance and $240 \mu \mathrm{m}$ maximum displacement of the centre point of the coil

\begin{tabular}{llllll}
\hline Model & $\begin{array}{l}f_{\text {res }} \\
(\mathrm{kHz})\end{array}$ & $\begin{array}{l}\text { Beam width } \\
(\mu \mathrm{m})\end{array}$ & $\begin{array}{l}R_{\text {load }} \\
(\mathrm{k} \Omega)\end{array}$ & $\begin{array}{l}V_{\text {load }} \\
(\mathrm{V})\end{array}$ & $\begin{array}{l}P_{\text {load }} \\
(\mathrm{mW})\end{array}$ \\
\hline $\mathrm{A}$ & 9.812 & 500 & 20.4 & 9.0 & 2.0 \\
$\mathrm{~B}$ & 7.149 & 400 & 14.8 & 6.5 & 1.45 \\
$\mathrm{C}$ & 4.743 & 300 & 9.8 & 4.3 & 0.96 \\
\hline
\end{tabular}

The amplitude of vibration is limited by the surrounding silicon frame thereby providing physical overrange protection. The corresponding maximum displacement of the centre point of the coil is $240 \mu \mathrm{m}$. The reduced stiffness of the lower frequency designs means that the level of stress experienced at the maximum deflection is lower than for the stiffer structures. In the case of device $C$, the least stiff device, the maximum stress is $2.6 \mathrm{GPa}$ which is well below the yield strength of silicon. The ultimate robustness of the generator will in practice be limited by surface and/or material imperfections and any impact stress arising from the paddle contacting the frame. The influence of surface roughness is discussed in more detail in the fabrication section below.

\section{Magnetic modelling}

Electromagnetic FEA simulations using Ansoft's Maxwell 2D software has been performed to determine the magnitude of the voltage which can be generated from the single beam generator designs. The coil used in the initial simulation is a conventionally wound enamelled copper coil with 600 turns of $25 \mu \mathrm{m}$ wire. The outer diameter of the coil is $2.4 \mathrm{~mm}$ and the inner diameter is $0.6 \mathrm{~mm}$. The separation between magnets and coil is $0.1 \mathrm{~mm}$. The resulting mass of the silicon paddle plus coil is measured to be $2.8 \times 10^{-5} \mathrm{~kg}$. As described above, and shown in Fig. 1, the coil is positioned between the magnets. Four magnets are arranged in pairs to form a two-pole arrangement as shown in Fig. 4. The magnets used were sintered $\mathrm{NdFeB}$ with dimensions of $1 \times 1 \times 3 \mathrm{~mm}$. 3D FEA simulation has been used to verify the flux density distribution between the magnets. The flux density distribution for the two-pole configuration is shown in Fig. 5. As can be seen from the plot the flux density varies from approximately $+0.5 \mathrm{~T}$ under one pole to approximately $-0.5 \mathrm{~T}$ under the other pole. In order to determine the voltage which would be induced in the coil, given a certain vibration frequency, $f$, coil displacement, $X_{\max }$, and velocity, $v$, a 2D transient finite element simulation is used. A sinusoidal velocity given by $v=X_{\max } 2 \pi f \sin (2 \pi f t)$, is used as input to the transient simulation to specify the coil movement.

Table 1 gives the predicted results for the voltages and powers generated according to the FEA for the three 
Fig. 3 Generator mode of operation
1

NODAL SOLUTION

NN

STEP-1

SUB -1

TIMB - 1

81

(AVG)

DIII $=-.55803$

$\operatorname{SMX}=.264 \mathrm{~B}+10$
MaR 242004

$11: 22 ; 00$
Magnets
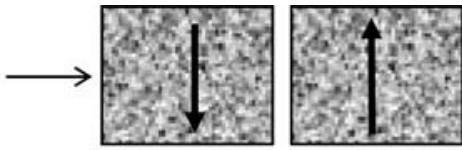

Coil

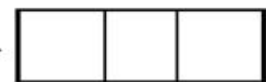

Field direction

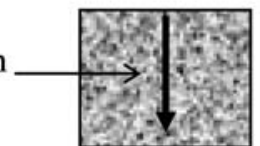

Fig. 4 Magnet configuration

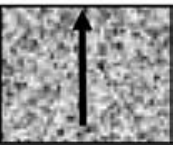

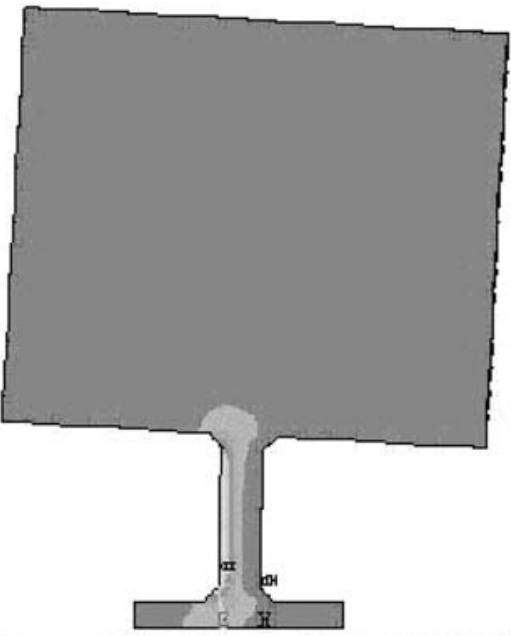

\section{$-.488 \mathrm{E}+09 \cdot-.141 \mathrm{~B}+09^{.07 \mathrm{E}+09} \cdot .554 \mathrm{~B}+09^{.902 \mathrm{E}+09^{2}} \cdot 125 \mathrm{~B}+10^{.160 \mathrm{E}+10} \cdot 194 \mathrm{~B}+10^{.829 \mathrm{E}+10} \cdot 264 \mathrm{~B}+10$ si11con M1crogenerator}

Fig. 5 3D FEA simulation of flux density for the two-pole configuration with $100 \mu \mathrm{m}$ separation distance between coil and permanent magnets

beam geometries $A, B$ and $C$ and two-pole magnet configuration for a $240 \mu \mathrm{m}$ coil displacement and a $100 \mu \mathrm{m}$ separation distance between the coil and the permanent magnets. The simulation results indicate that sufficient high voltages, which allow rectification without significant loss, can be generated. The estimated power is based on the power delivered to the load resistance indicated in the table. This value of load resistance has been chosen so as to maximise the displacement for $1 g$ vibration acceleration as described below.

For the purposes of the 2D finite element simulation the maximum displacement is fixed at $240 \mu \mathrm{m}$ and it is

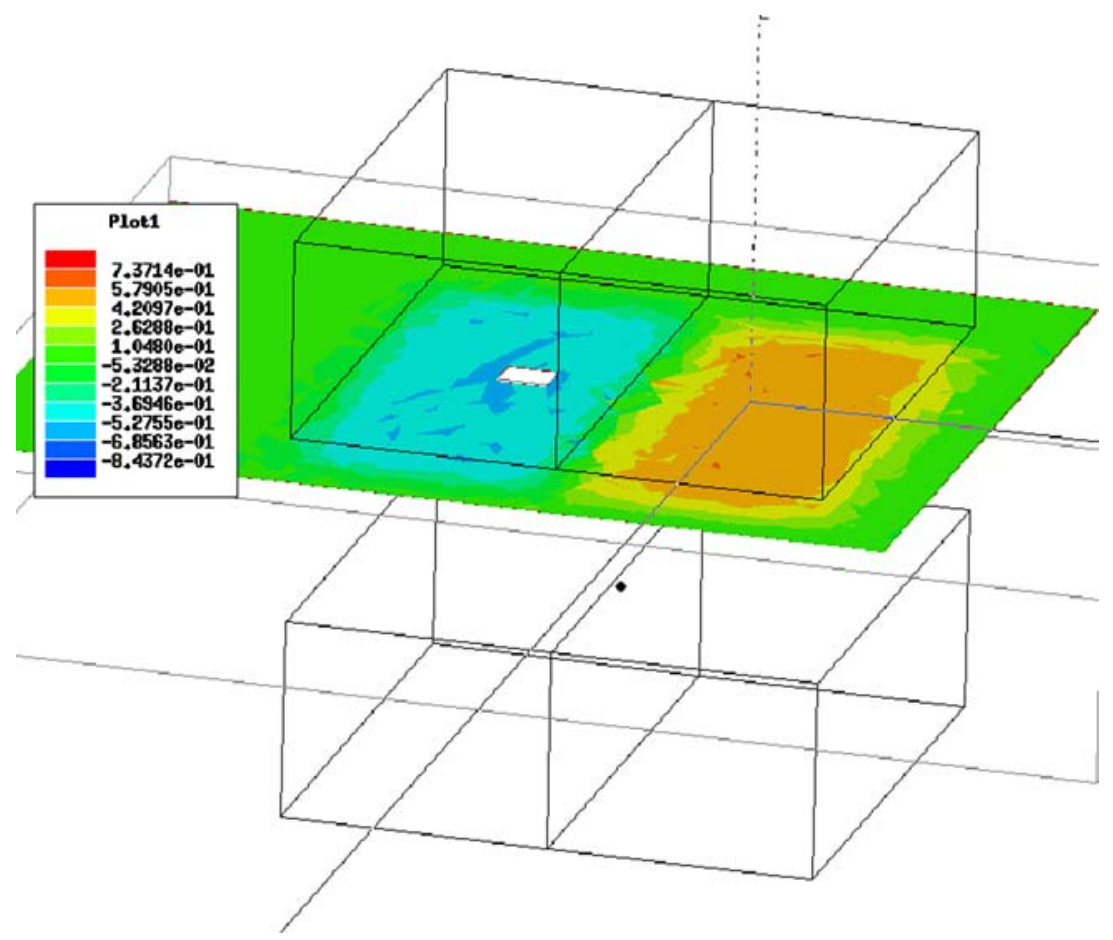


assumed that sufficient mechanical force, given by the product of mass and acceleration, exists to give the required displacement. In order to illustrate how this is affected by the choice of load resistance, we assume that the motion of the device can be described as a single degree of freedom, damped mass-spring system, as described in $[2,4,5]$. If we assume that the device is operated at resonance, i.e. the frequency of the driving vibration is equal to the natural frequency of the device, then it can be shown that the amplitude of the movement of the mass is given by [4];

$X_{\max }=\frac{m a}{D 2 \pi f_{\text {res }}}$

where, $D$ is the electromagnetic damping factor for the device, and $m$ is the moving mass, and $a$ is the acceleration of the input vibration. In this case only electromagnetic damping is considered, and the electromagnetic damping factor, $D$ can be estimated by [4]

$$
D=\frac{(N l B)^{2}}{R_{\text {load }}+R_{\text {coil }}+j \omega L_{\text {coil }}}
$$

where $N$ is the number of turns in the generator coil, $l$ is the side length of the coil (assumed square), and $B$ is the flux density to which it is subjected and $R_{\text {load }}, R_{\text {coil }}$, and $L_{\text {coil }}$ are the load resistance, coil resistance and coil inductance, respectively. Equation 2 shows that the damping factor can be adjusted by varying the load resistance for a given device since all the other parameters are fixed by the geometry. The expression in Eq. 1 shows that the maximum displacement is inversely proportional to the damping factor, and proportional to the input acceleration with the other parameters fixed by the device geometry. Provided, as it usual, the electromagnetic damping is dominant. If the input acceleration is fixed at a value of $1 \mathrm{~g}\left(9.81 \mathrm{~m} \mathrm{~s}^{-2}\right)$, then the maximum displacement can be controlled by choosing the value of load resistance, $R_{\text {load }}$. The value of load resistance shown in Table 1 corresponds to the value required to achieve a $240 \mu \mathrm{m}$ displacement of the paddle for $1 \mathrm{~g}$ input acceleration.

After assembly, the devices were inspected and some deviations from the simulated parameters were observed. In the fabricated devices the separation between the magnet and coil is $0.5 \mathrm{~mm}$ as opposed to the $0.1 \mathrm{~mm}$ separation assumed above. This was due to the use of a Perspex chip, which was glued to the generator chip as described in Sect. 5. This increase in separation between coil and magnets will result in a decrease in the flux density between the magnets. In order to compensate in part for this, a mild steel yoke has been used across the magnets. The diagram in Fig. 6 shows the half model of the actual device which has been fabricated. Figure 7 shows the plot of flux density between the magnets for this configuration. The flux density varies from $+0.2 \mathrm{~T}$ under one magnet to $-0.2 \mathrm{~T}$ under the other. This is significantly less than $0.5 \mathrm{~T}$ predicted for the closer separation in the earlier simulations. In addition to these changes, the actual coils used only have 600 turns due to a lower than expected coil filling factor. The measured resistance of the coil is $112 \Omega$ and the inductance is $367 \mu \mathrm{H}$ at $10 \mathrm{kHz}$. From experimental tests on the devices, the resonant frequencies were found to be 1.6 and $9.5 \mathrm{kHz}$. Table 2 shows the predicted voltages from the actual devices, assuming that the full paddle displacement of $240 \mu \mathrm{m}$ is achieved. The table also gives the predicted power delivered to a $2 \mathrm{k} \Omega$ load resistance.

\section{Micro generator fabrication}

The prototype uses discrete components for the permanent magnets and coil, and employs seven masks in the fabrication process. The full device consists of three wafers bonded together. The central silicon wafer was etched by DRIE to fabricate the silicon paddle, frame and beam. A $\mathrm{KOH}$ etched $\mathrm{V}$ groove is also included along the centre line of the beam to provide a channel for the copper wire from the coil. In this way the wires can be kept below the surface of the wafer and not interfere with bonding. The full batch fabrication process is shown in Fig. 8. The capping wafers will eventually be made from Pyrex wafers with etched recesses for location of the permanent magnets and also to provide clearance for the paddle to vibrate. These Pyrex wafers will be anodically bonded to each face of the silicon wafer. However, the initial assemblies use Perspex chips of similar dimensions fabricated using traditional milling techniques. The Perspex chips were individually glued to the silicon chips during assembly.

A silicon paddle with a $500 \mu \mathrm{m}$ wide beam, v-groove and frame for the prototype microgenerator is shown in the scanning electron microscope (SEM) image in Fig. 9. Figure 10 shows the components of the generator prior to assembly. The centre chip shows the coil integrated within the silicon paddle. The two permanent NdFeB magnet pairs can be seen mounted in the outer Perspex

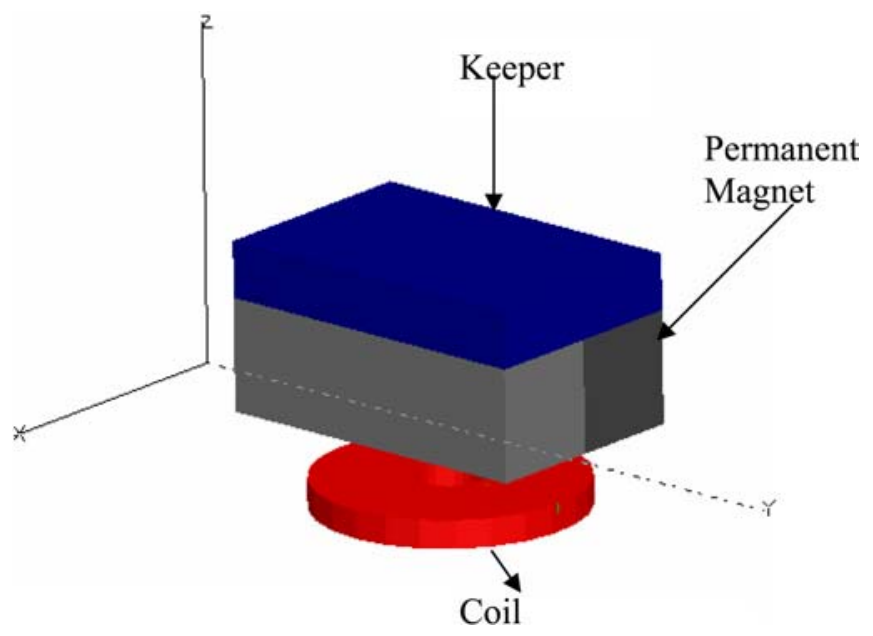

Fig. 6 Magnet configuration used in the simulation and the half model used for the 3D simulation 
Fig. 7 3D FEA simulation of flux density for the two-pole configuration with $500 \mu \mathrm{m}$ separation distance between coil and permanent magnets

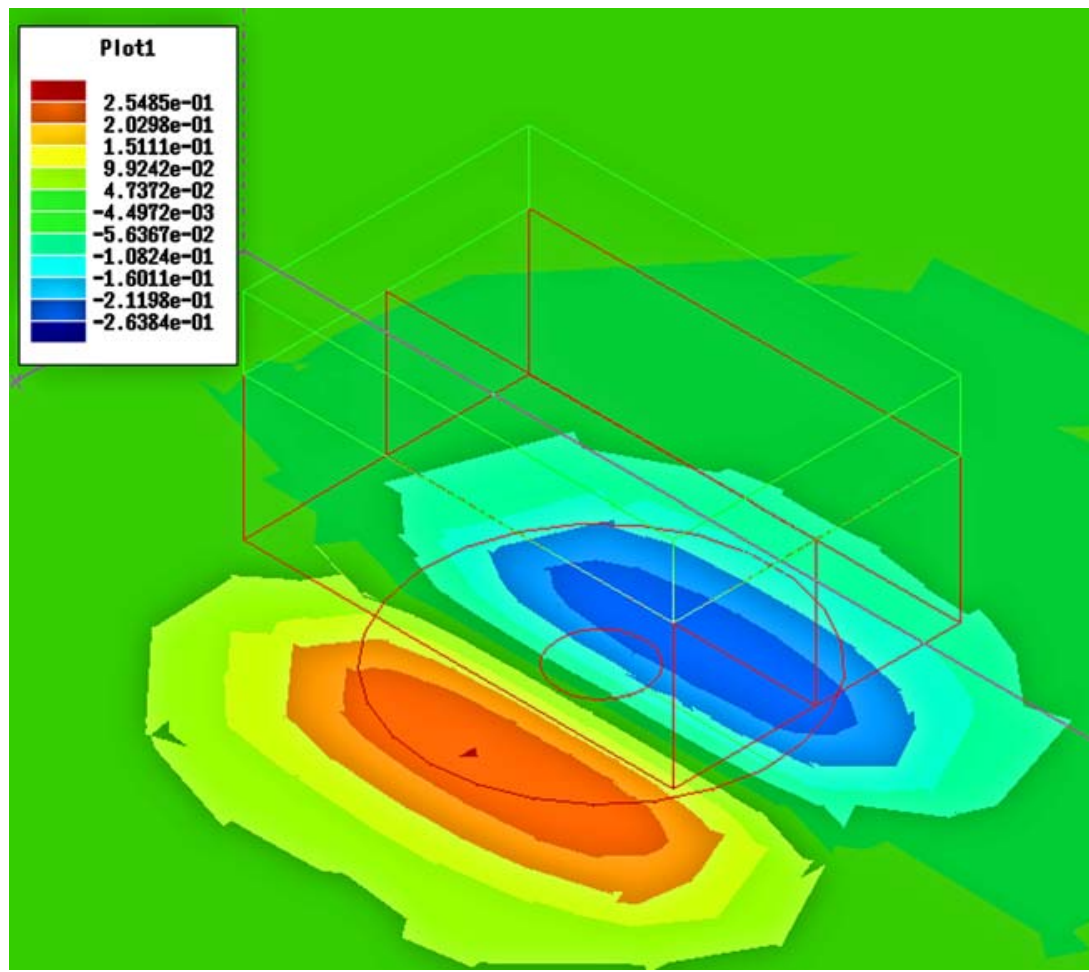

Table 2 Voltages and power obtained from the 2D transient simulation for two-pole configuration with $500 \mu \mathrm{m}$ separation distance and $240 \mu \mathrm{m}$ maximum displacement of the centre point of the coil

\begin{tabular}{lll}
\hline $\begin{array}{l}\text { Frequency } \\
(\mathrm{kHz})\end{array}$ & $\begin{array}{l}\text { Peak } \\
\text { voltage }(\mathrm{V})\end{array}$ & $\begin{array}{l}\text { Power } 2 \mathrm{k} \Omega \\
\text { load }(\mathrm{mW})\end{array}$ \\
\hline 1.6 & 0.7 & 0.11 \\
9.5 & 4.15 & 3.35 \\
\hline
\end{tabular}

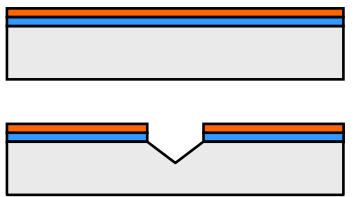

$1 \mathrm{~m} \mathrm{SiO}_{2}$

$160 \mathrm{~nm} \mathrm{Si}_{3} \mathrm{~N}_{4}$

Photolith trench, Dry etch $\mathrm{SiO}_{2} / \mathrm{Si}_{3} \mathrm{~N}_{4}$ Wet $\mathrm{KOH}$ etch $\mathrm{Si}$

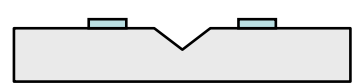
Strip $\mathrm{SiO}_{2} / \mathrm{Si}_{3} \mathrm{~N}_{4}$ Evaporate $1 \mathrm{~m} \mathrm{Al}$ Photolith and wet etch Al

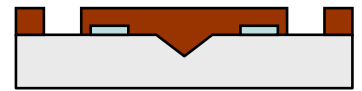

Photolith Si paddle SPR220 resist

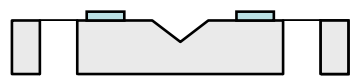

DRIE Si through wafer

Strip resist

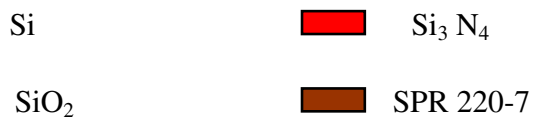

Fig. 8 Discrete coil generator fabrication process chips. Figure 11 shows an assembled microgenerator mounted into a standard package.

\section{Testing results}

In order to evaluate the output power from the microgenerators, devices were tested using a Bruel \& Kjaer type 4810 mini shaker driven from a signal generator via a $100 \mathrm{~W}$ RMS MOSFET amplifier. Excitation vibrations were measured using a PCB Piezotronics model

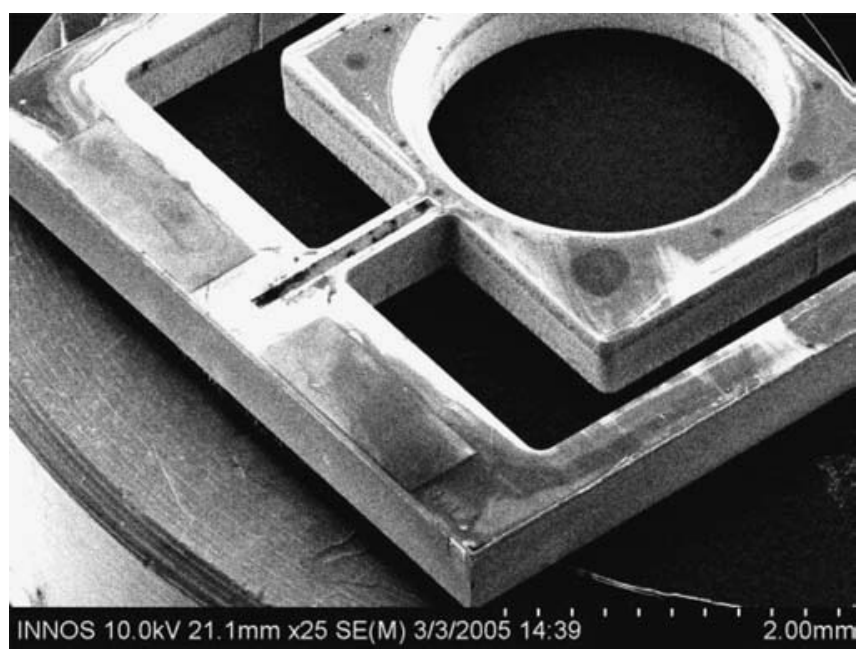

Fig. 9 SEM image of Silicon paddle chip with v-groove 
Fig. 10 Generator components (two Perspex chips with magnets and silicon paddle and coil)
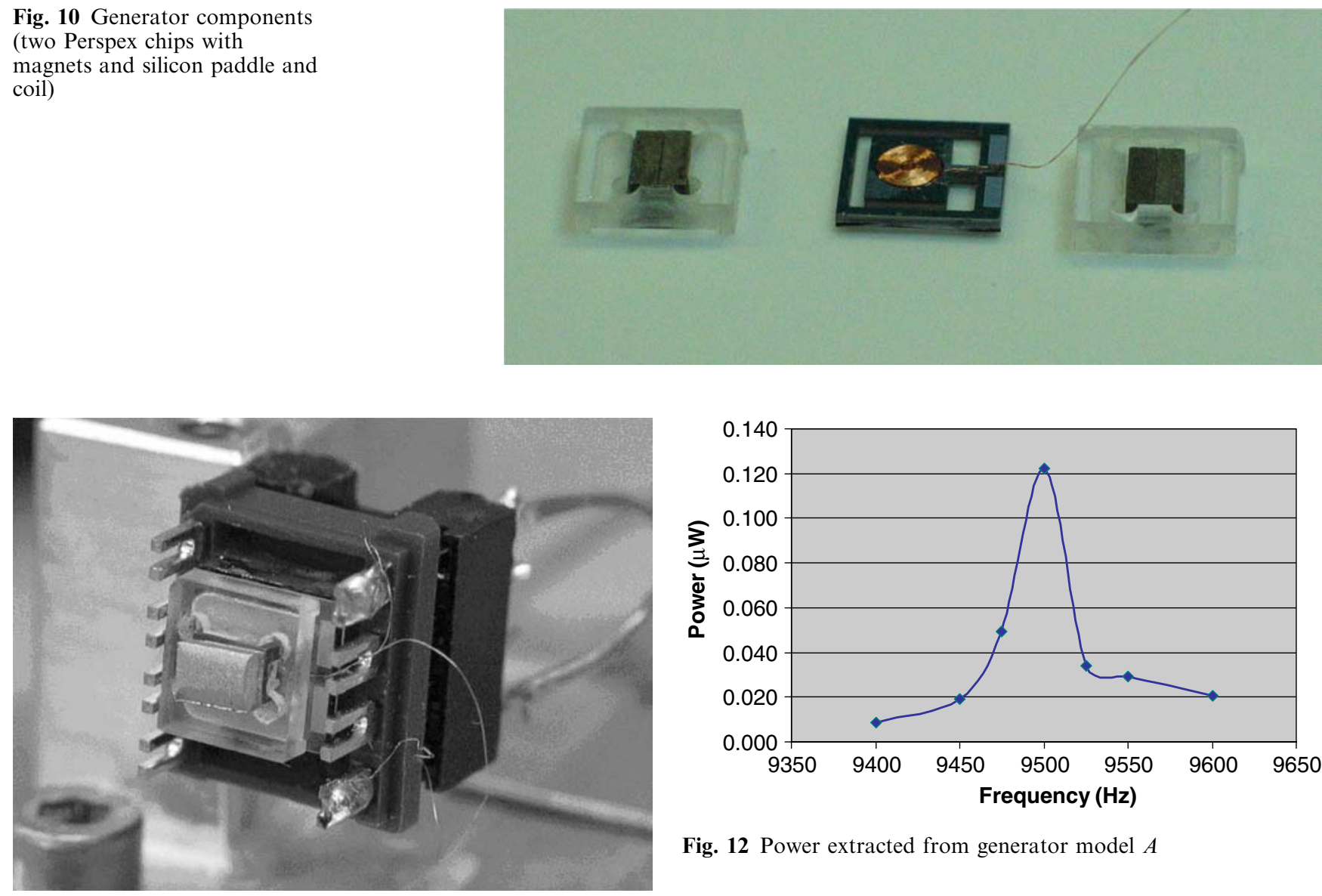

Fig. 12 Power extracted from generator model $A$

Fig. 11 Assembled microgenerator

$354 \mathrm{C} 03$ three-axis accelerometer. The tests were performed at acceleration levels of $0.4 \mathrm{~g}\left(3.9 \mathrm{~m} \mathrm{~s}^{-2}\right)$ and the resonant frequency of the generator observed on a Hewlett Packard 3588 A spectrum analyser. The output of the generator was applied across an impedance-matched load and the voltage observed on a digital oscilloscope. Typical generator outputs are shown in Fig. 12. Figure 12 shows the results from a model A cantilever generator with a beam width of $500 \mu \mathrm{m}$. This was found to have a resonant frequency of $9.5 \mathrm{kHz}$ and generated $122 \mathrm{nW}$ into a $100 \Omega$ load with a $Q$-factor of approximately 200. The difference between the measured and simulated resonant frequencies is predominantly due to the influence of the assembly process and the bonding of the enamelled copper wires along the beam or across the meander. It is also clear from the measured results that mechanical damping mechanisms are dominating. These undesirable damping effects need to be substantially reduced in order to achieve the predicted power levels given in Table 2.

\section{Conclusions}

This work presents the mechanical and electromagnetic behaviour of a silicon vibration-powered generator.
Different beam dimensions have been investigated. Magnetic finite element simulations predict that voltage levels of $0.7-4.15 \mathrm{~V}$ can be generated for a full $240 \mu \mathrm{m}$ displacement of the paddle. Simulations show these voltages can be increased if the separation between the magnets and moving coil is decreased to $0.1 \mathrm{~mm}$ and the number of turns in the coil is increased. With these improvements FEA predicts that voltages between 4 and $9 \mathrm{~V}$ can be generated for a $1 g$ acceleration input, with an optimised choice of load resistance. Further work to improve the performance of the generators by reducing the gap between the magnets and the coil and reducing the magnitude of undesirable damping effects is currently underway. This will be achieved by optimising fabrication and assembly process and operating the devices in a vacuum.

Acknowledgements The authors would like to acknowledge the European Union for funding this Framework 6 STREP project VIBES, project reference 507911 .

\section{References}

1. Najafi K (2000) Low-power micromachined Microsystems. In: IEEE proceedings. 1st International symposium on low power electronics and design, pp 1-8

2. Shearwood C, Yates RB (1997) Development of an electromagnetic micro-generator. Electron Lett 33(22):1883-1884 
3. Ching NNH, Wong HY, Li WJ, Leong PHW, Wen Z (2002) A laser-micromachined multi-modal resonating power transducer for wireless sensing systems. Sens Actuators A Phys 97-98:685-690

4. El-Hami M, Glynne-Jones P, James E, Beeby SP, White NM, Brown AD, Ross JN, Hill M (2001) Design and fabrication of a new vibration-based electromechanical power generator. Sens Actuators A Phys 92:335-342
5. Mitcheson PD, Green TC, Yeatman EM, Holmes AS (2004) Architectures for vibration-driven micropower generators. J MEMS 13(3):429-440

6. Glynne-Jones P, Tudor MJ, Beeby SP, White NM (2004) An electromagnetic, vibration-powered generator for intelligent sensor systems. Sens Actuators A Phys 110(1-3):344-349 